\title{
Sudden Cardiac Death Prediction in Chronic Heart Failure Patients by Periodic Repolarization Dynamics
}

\author{
Saúl Palacios ${ }^{1}$, Iwona Cygankiewicz ${ }^{2}$, Antoni Bayés-de-Luna ${ }^{3}$, Juan Pablo Martínez ${ }^{1,4}$ and Esther \\ Pueyo $^{1,4}$ \\ ${ }^{1}$ BSICoS, Aragon Institute of Engineering Research, IIS Aragón, Universidad de Zaragoza, \\ Zaragoza, Spain \\ ${ }^{2}$ Department of Electrocardiology, Medical University of Lodz, Lodz, Poland \\ ${ }^{3}$ Institut Català de Ciències Cardiovasculars, Santa Creu i Sant Pau Hospital, Barcelona, Spain \\ ${ }^{4}$ CIBER en Bioingeniería, Biomateriales y Nanomedicina (CIBER-BBN), Spain
}

\begin{abstract}
Chronic heart failure (CHF) is a clinical syndrome associated with high mortality due to pump failure death (PFD) resulting from heart failure progression as well as to ventricular arrhythmias leading to sudden cardiac death (SCD). CHF involves autonomic nervous system imbalance, which is expected to be reflected in the electrocardiogram (ECG). Periodic Repolarization Dynamics (PRD) quantifies low-frequency oscillations in the $T$ wave of the ECG and has been related to sympathetic modulation of ventricular repolarization. We assessed the capacity of PRD to predict PFD and SCD in a CHF population. 3-lead ECG recordings of 569 patients with symptomatic $C H F$ were analyzed. PRD values were measured by analyzing 5-minute segments with 4-minute overlap. The minimum PRD value over the analyzed segments was assigned to each patient. PRD was higher in SCD victims than in PFD victims and than in survivors and non-cardiac death victims, although differences were not statistically significant. Low- and high-risk groups were defined by dichotomization according to median PRD in the population. Hazard ratio [95\% Confidence Interval] for SCD from univariate Cox regression was 1.808 [1.031-3.169] deg $(p=0.039)$. In conclusion, high PRD predicts $S C D$ in a CHF population, with SCD victims presenting enhanced sympatheticinduced oscillations of ventricular repolarization.
\end{abstract}

\section{Introduction}

Chronic heart failure (CHF) is a cardiac syndrome with high morbidity and mortality rates worldwide. Only in the United States, there are currently around 6 million people suffering from $\mathrm{CHF}$ and projections indicate that more than 8 million people will suffer from this condition by 2030 [1]. CHF is characterized by functional and struc- tural abnormalities in the heart that hamper its ability to pump blood sufficiently to maintain the body's needs. Importantly, autonomic imbalance has been shown to occur in CHF, with increased sympathetic activity and withdrawal of parasympathetic activity, altogether leading to further worsening of CHF prognosis [2].

Sudden cardiac death (SCD), defined as unexpected death caused by loss of heart function, represents the main underlying cause of mortality in CHF patients. Another common cause of death in CHF is pump failure death (PFD), resulting from a deterioration of heart failure. Prediction of these two modes of death is of major importance, as it can help in the application of more tailored therapies, which can lead to an improvement in the quality of life of affected patients.

A number of non-invasive markers measured from the electrocardiogram (ECG) have been proposed for PFD and SCD risk stratification in CHF. Among others, QT dispersion [3], Tpeak-to-Tend interval [4] and $\mathrm{T}$ wave alternans [5] have been evaluated. Periodic Repolarization Dynamics (PRD) has been recently proposed to assess sympathetic modulation of ventricular repolarization by measuring low-frequency (below $0.1 \mathrm{~Hz}$ ) oscillations in the $\mathrm{T}$ wave vector [6]. The capacity of PRD to predict total and arrhythmic mortality and to identify benefit from ICD implantation in post-myocardial infarction populations has been demonstrated $[6,7]$. The aim of this work is to assess the capacity of PRD to risk stratify for PFD and SCD in a cohort of $\mathrm{CHF}$ patients.

\section{Study Population}

The study population consisted of 569 patients with symptomatic CHF, New York Heart Association (NYHA) classes II and III, enrolled in the MUSIC (MUerte Subita en Insuficiencia Cardiaca) study, a prospective, multicen- 
ter, longitudinal study designed to assess risk predictors of cardiac mortality and SCD in CHF ambulatory patients [8].

20-minute high-resolution $(1000 \mathrm{~Hz})$ ECG recordings were acquired at rest by using SpiderView recorders (ELA Medical, SorinGroup, Paris, France). Three orthogonal leads were analyzed for each patient.

Patients were followed up every 6 months on an outpatient basis for an average of 48 months. The study group included 53 victims of SCD, 53 victims of PFD, 37 noncardiac death victims and 426 survivors. Death was defined as SCD if it was: (i) a witnessed death occurring within 60 minutes from the onset of new symptoms, unless a cause other than cardiac was obvious; (ii) an unwitnessed death ( $<24$ hours) in the absence of preexisting progressive circulatory failure or other causes of death; or (iii) a death during attempted resuscitation. Endpoints were reviewed and classified by the MUSIC Study Endpoint Committee. The study protocol was approved by institutional investigator committees and all patients gave written informed consent.

\section{Methods}

\subsection{Preprocessing}

Preprocessing of ECG signals included noise removal using a $50 \mathrm{~Hz}$ notch filter, heart beat detection and labelling using Aristotle ECG analysis software [9], and these signals were postprocessed applying the method described in [10]. Since subsequent analysis focused on the $\mathrm{T}$ wave, a $40 \mathrm{~Hz}$ low-pass filter was applied to remove noise without altering the $\mathrm{T}$ wave shape. Finally, cubic splines interpolation was applied to estimate and remove baseline wander.

A window including the $\mathrm{T}$ wave was defined from $\mathrm{T}$ wave onset, $\mathrm{T}_{\mathrm{on}_{i}}$, to $\mathrm{T}$ wave end, $\mathrm{T}_{\text {off }_{i}}$, defined according to the following rules, expressed as a function of the corresponding $\mathrm{QRS}$ mark $\left(\mathrm{QRS}_{i}\right)$ and $\mathrm{RR}$ interval $\left(\mathrm{RR}_{i}\right)$, with $i$ denoting beat index:

- If $\mathrm{RR}_{i}<720 \mathrm{~ms}$,

$$
\begin{gathered}
\mathrm{T}_{\mathrm{on}_{i}}=\mathrm{QRS}_{i}+90 \mathrm{~ms} \\
\mathrm{~T}_{\text {off }_{i}}=\mathrm{QRS}_{i}+\min \left(360, \frac{2}{3} \mathrm{RR}_{i}\right) \mathrm{ms} .
\end{gathered}
$$

- If $\mathrm{RR}_{i} \geq 720 \mathrm{~ms}$,

$$
\begin{gathered}
\mathrm{T}_{\mathrm{on}_{i}}=\mathrm{QRS}_{i}+90 \mathrm{~ms} \\
\mathrm{~T}_{\text {off }_{i}}=\mathrm{QRS}_{i}+360 \mathrm{~ms} .
\end{gathered}
$$

A noise level was defined for each $\mathrm{T}$ wave by measuring the high-frequency components (above $15 \mathrm{~Hz}$ ) [11]. The defined $\mathrm{T}$ wave window boundaries were discarded for low-quality beats (noise level $>140 \mu \mathrm{V}$ ) but the associated QRS marks were retained.

\subsection{Periodic Repolarization Dynamics}

The angles between consecutive $\mathrm{T}$ waves were calculated from the preprocessed ECG signals by an updated method, defined in [12], based on the original method proposed in [6]. The method included the following steps:

1. The boundaries of each $\mathrm{T}$ wave window were identified according to the method described in section 3.1.

2. A constant value was subtracted from each $\mathrm{T}$ wave in each of the analyzed leads so that the amplitude at the $\mathrm{T}$ wave end boundary was set to $0 \mathrm{mV}$.

3. An average $\mathrm{T}$ wave vector was calculated for each $\mathrm{T}$ wave window. The angle $\mathrm{dT}^{\circ}$ between two consecutive $\mathrm{T}$ waves, which is associated with the instantaneous degree of repolarization instability, was calculated by using the dot product of the two average $\mathrm{T}$ wave vectors.

4. A 10th-order median filter was applied to the $\mathrm{dT}^{\circ}$ time series to attenuate outliers and avoid very abrupt changes.

To compute PRD from the $\mathrm{dT}^{\circ}$ series, a phase-rectified signal averaging (PRSA)-based method was used [13], which included:

5. Anchor points were defined by comparing averages of $M=9$ values of the $\mathrm{dT}^{\circ}$ series prior and posterior to each anchor point candidate $x_{i}$. A beat $i$ was considered an anchor point if:

$$
\frac{1}{M} \sum_{j=0}^{M-1} x_{i+j}>\frac{1}{M} \sum_{j=1}^{M} x_{i-j} .
$$

The use of $M=9$ allows detecting frequencies in the range of interest (from 0.025 to $0.1 \mathrm{~Hz}$ ).

6. Windows of $W=40$ values were defined around each anchor point. If an anchor point was so close to the beginning or to the end of the $\mathrm{dT}^{\circ}$ series that there were not enough samples before or after it, it was disregarded.

7. PRSA series was obtained by averaging the $\mathrm{dT}^{\circ}$ series over all defined windows of length $W$ described in the previous step.

PRD was defined as the difference between the maximum and minimum values of the PRSA series. Each 20-minute ECG recording was divided into 5-minute segments with 4-minute overlap and PRD was measured in each of those segments. Finally, each patient was assigned with the minimum PRD value over segments.

\subsection{Statistical analysis}

PRD values in patient subgroups are presented as median [interquartile range (IQR)]. The Mann-Whitney U test (or Wilcoxon rank-sum test) was used for univariate comparisons of PRD values between patient groups. Survival probability was estimated and graphically shown according to the Kaplan-Meier method, with comparisons between survival times performed by the log-rank test. The 
Cox proportional hazards regression model was used in the prediction of SCD, with hazard ratios (HAR) and 95\% confidence intervals used for characterization. Statistical differences are considered when significance level is $\mathrm{p}$ $<0.05$. Variables providing significantly different survival times between groups were entered into the Cox regression model. MATLAB R2017a (9.2) and SPSS (version 24.0) were used for the analysis.

\section{Results}

\subsection{Association with cardiac mortality}

Figure 1 presents boxplots showing statistical distributions of PRD in three analyzed patient subgroups, namely SCD victims, PFD victims and the rest of patients (survivors plus non-cardiac death victims). Although no significant differences were found between these groups, PRD showed a trend towards higher values in SCD victims (with median [IQR] being 1.57 [1.21] deg) than in PFD victims (1.41 [0.91]) and even more than in survivors and non-cardiac death victims (1.24 [1.28] deg).

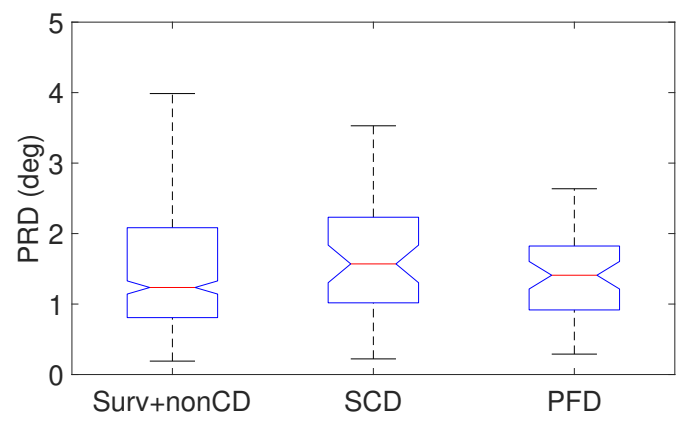

Figure 1. Boxplots of PRD for three patient subgroups: SCD, PFD and the rest of patients (survivors plus noncardiac death victims).

\subsection{Survival analysis}

The median PRD value in the study population was used to define low-risk (PRD $<1.31 \mathrm{deg}$ ) and high-risk (PRD $\geq$ $1.31 \mathrm{deg}$ ) groups. When PFD was considered as endpoint, no significant differences in the survival times were found between those two groups. For SCD as endpoint, differences between survival times in the low-risk and high-risk groups were statistically significant $(p=0.036)$. The probability curves for survival to SCD along follow-up are presented in Figure 2.

In univariate Cox proportional model using PRD as the predictor variable, the hazard ratio [95\% confidence interval] for SCD was 1.808 (1.031-3.169), $p=0.039$.

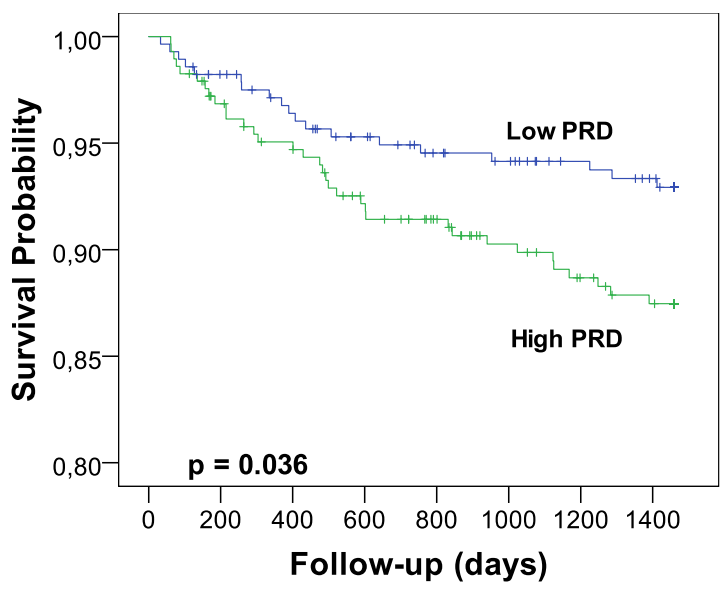

Figure 2. Estimated probability of survival to SCD for two patient subgroups dichotomized according to PRD.

\section{Discussion and conclusions}

Low-frequency oscillations in the $\mathrm{T}$ wave of the ECG were non-invasively measured by quantifying the PRD marker in 20-minute ECG recordings of CHF patients. PRD has been associated with sympathetic modulation of ventricular electrical activity [6]. Clinical, experimental and theoretical studies have shown that sympathetic provocation leads to increased magnitude of low-frequency oscillations of ventricular repolarization as measured from the $\mathrm{T}$ wave of the ECG, from activation recovery intervals of electrograms and from cellular action potentials [7, 14-16]. Potential mechanisms underlying those sympathetic-induced increases have been postulated, involving synergistic $\beta$-adrenergic and mechanical effects $[14,17]$.

When investigating PRD values separately in PFD victims, SCD victims and the rest of patients, differences were found, even if not reaching statistical significance. The highest PRD values were measured in SCD victims, which could possibly be indicative of higher sympathetic modulation of repolarization in those patients. After dichotomizing the study population into low- and high-risk groups based on median PRD, we found that SCD probability during follow-up was almost double in the high-risk than in the low-risk group ( $p=0.036)$. This is in agreement with clinical and theoretical studies showing a relationship between increased PRD and enhanced arrhythmic risk $[6,7,14,17]$.

An interesting feature of this research is that PRD was calculated in 20-minute resting ECG recordings, thus facilitating generalization of PRD evaluation for risk stratification purposes. Other ECG indices, such as heart rate turbulence, deceleration capacity [18] or microvolt T wave alternans [5] are commonly measured from ambulatory ECG 
recordings or using specific protocols like stress tests.

In conclusion, this study indicates that PRD, a noninvasive marker of repolarization instability measuring low-frequency $\mathrm{T}$ wave oscillations, has the potential to predict SCD in CHF from short resting ECG recordings, with higher PRD values indicative of higher SCD risk.

\section{Acknowledgements}

This work was supported by projects ERC-StG 638284 (European Research Council), PID2019-105674RB-I00 and PID2019-104881RB-I00 (Ministerio de Ciencia e Innovación) and project LMP124-18 and reference group BSICoS T39-20R (Aragón Government cofunded by FEDER 2014-2020 "Building Europe from Aragon"). Computations were performed using ICTS NANBIOSIS (HPC Unit at University of Zaragoza).

\section{References}

[1] Savarese G, Lund LH. Global public health burden of heart failure. Card Fail Rev. 2017; 3:7-11.

[2] Kishi T. Heart failure as an autonomic nervous system dysfunction. J Cardiol. 2012; 59:117-122.

[3] Galinier M, Vialette JC, Fourcade J, Cabrol P, Dongay B, Massabuau P, et al. QT interval dispersion as a predictor of arrhythmic events in congestive heart failure: Importance of aetiology. Eur Heart J. 1998; 19:1054-1062.

[4] Chua KCM, Rusinaru C, Reinier K, Uy-Evanado A, Chugh $\mathrm{H}$, Gunson K, et al. Tpeak-to-Tend interval corrected for heart rate: A more precise measure of increased sudden death risk? Heart Rhythm. 2016; 13:2181-2185.

[5] Daniłowicz-Szymanowicz L, Kaufmann D, Rozwadowska K, Kempa M, Lewicka E, Raczak G. Microvolt T-wave alternans and autonomic nervous system parameters can be helpful in the identification of low-arrhythmic risk patients with ischemic left ventricular systolic dysfunction. PLOS ONE. 2018; 13:1-15.

[6] Rizas KD, Nieminen T, Barthel P, Zürn C, Kähönen M, Viik $\mathrm{J}$, et al. Sympathetic activity-associated periodic repolarization dynamics predict mortality following myocardial infarction. J Clin Invest. 2014; 124(4):1770-1780.

[7] Rizas KD, McNitt S, Hamm W, Massberg S, Kääb S, Zareba W, et al. Prediction of sudden and non-sudden cardiac death in post-infarction patients with reduced left ventricular ejection fraction by periodic repolarization dynamics: MADIT-II substudy. Eur Heart J. 2017;38(27):21102118.

[8] Vazquez R, Bayes-Genis A, Cygankiewicz I, Pascual-Figal $\mathrm{D}$, Grigorian-Shamagian L, Pavon R et al. The MUSIC risk score: A simple method for predicting mortality in ambula- tory patients with chronic heart failure. Eur Heart J. 2009; 30:1088-1096.

[9] Moody GB, Mark RG. Development and evaluation of a 2-lead ECG analysis program. Computers in Cardiology. 1982; 9:39-44.

[10] Mateo J, Laguna P. Analysis of heart rate variability in the presence of ectopic beats using the heart timing signal. IEEE Trans Biomed Eng. 2003; 50:334-343.

[11] Mincholé A, Pueyo E, Rodríguez JF, Zacur E, Doblaré M, Laguna P. Quantification of restitution dispersion from the dynamic changes of the T-wave peak to end, measured at the surface ECG. IEEE Trans Biomed Eng. 2011; 58:11721182.

[12] Palacios S, Caiani EG, Landreani F, Martínez JP, Pueyo E. Long-term microgravity exposure increases ECG repolarization instability manifested by low-frequency oscillations of T-wave vector. Front Physiol. 2019; 10:1510.

[13] Bauer A, Kantelhardt JW, Bunde A, Barthel P, Schneider $\mathrm{R}$, Malik $\mathrm{M}$ et al. Phase-rectified signal averaging detects quasi-periodicities in non-stationary data. Physica A. 2006; 364:423-434

[14] Pueyo E, Orini M, Rodriguez JF, Taggart P. Interactive effect of beta-adrenergic stimulation and mechanical stretch on low-frequency oscillations of ventricular action potential duration in humans. J Mol Cell Cardiol. 2016; 97:93-105.

[15] Porter B, van Duijvenboden S, Bishop MJ, Orini M, Claridge S, Gould J et al. Beat-to-beat variability of ventricular action potential duration oscillates at low frequency during sympathetic provocation in humans. Front Physiol. 2018; 9:147.

[16] Duijvenboden SV, Porter B, Pueyo E, Sampedro-Puente DA, Fernandez-Bes J, Sidhu B, et al. Complex interaction between low-frequency apd oscillations and beat-to-beat apd variability in humans is governed by the sympathetic nervous system. Front Physiol. 2020; 10:1582.

[17] Sampedro-Puente DA, Fernandez-Bes J, Porter B, van Duijvenboden S, Taggart P, Pueyo E. Mechanisms underlying interactions between low-frequency oscillations and beatto-beat variability of celullar ventricular repolarization in response to sympathetic stimulation: Implications for arrhythmogenesis. Front Physiol. 2019; 10:916.

[18] Al-Zaiti SS, Pietrasik G, Carey MG, Alhamaydeh M, Canty JM, Fallavollita JA. The role of heart rate variability, heart rate turbulence, and deceleration capacity in predicting cause-specific mortality in chronic heart failure. J Electrocardiol. 2019; 52:70-74.

Address for correspondence:

Saúl Palacios

Lab 3.07, Edificio Ada Byron

María Luna 1, 50018 Zaragoza (Spain)

spalacios@unizar.es 